\title{
ADAPTIVE MORPHOLOGY SHORTEST PATH PLANNING BASED ON ROTATING OBJECT AND CRITICAL TRANSITION POINT
}

\author{
Soo-Chang Pei, and Ching-Long Tseng
}

Department of Electrical Engineering, National Taiwan University

No. 1 Sec. 4, Roosevelt Rd., Taipei, 10617, Taiwan, R. O. C.

Tel.: 886-2-23635251-321, FAX: 886-2-23671909

E-mail: pei@cc.ee.ntu.edu.tw, tcl@ntserver.ee.ntu.edu.tw

\begin{abstract}
This paper presents a general approach to find the shortest path for a general moving object with rotation and forward/backward movement among obstacles of arbitrary shapes. We call the object of finding a shortest path as path planning. By utilizing the concept of the critical transition point and adaptive path planning, finding a shortest path problem of car-like vehicles becomes more practical. Moreover, additional efficiency in routing can be btained since the critical transition point is used in the configuration space. Notice that the computational complexity is not increased. A car-like vehicle model with forward/backward driving and turning mechanism is used to validate the performance of the proposed algorithms. Simulation results show that not only the proposed algorithms are successful in various environments, but also these algorithms have good efficiency in computation. Moreover, routing is gained by the help of morphological theory.
\end{abstract}

Keywords: critical transition point, adaptive path planning, rotation morphology, MMDT.

\section{INTRODUCTION}

To find a shortest path (path planning) is major and has stimulated considerable interest in modern manufacturing and other high technology fields of robotics and artificial intelligence. In the workspace, let $B$ represents the moving object and pe-assigned orientation for $B$, to determine whether there is a continuous collision-free path from a starting point $Z_{1}$ to an end point $Z_{2}$ with some criteria such as minimal cost or constraints of $B$.

In general, these algorithms can be divided into two major classes, one is configuration space method [1], and the another is computational geometry method [2]. In a word, the configuration space method can be regarded as adding the geometry of the moving object around the obstacles. That is, in order to create a new space so as to make the vehicle to be viewed as a reference point, the geometry of the moving object, is essentially set theoretically added around the obstacles. Most the configuration space method has the advantage of easy implementation and less computational complexity, but cannot solve the complicated cases traditionally. Some approaches use the conventional morphology to obtain the configuration space for non-rotating objects [1], and recently rotational morphology is used to find the shortest path for rotating objects [3], [4].

Another approach is to search the original free space directly without transforming the workspace into configuration space by using computational geometry, for solving such kind of problem. Translations are performed along freeways with rotations performed at the intersections of freeway [2]. This approach is called computational geometry method. Most the computational geometry methods have the advantage of being able to solve complex cases so as to be more close to real world applications, but in creases computational complexity if obstacles gather closely. The causes intractable system sometimes [5], some limitations, such as polyhedral assumption etc., so must be added to simplify problem.

Up to now, a rotational morphological approach is recently proposed to solve the path planning problem [3], thus the compur tational complexity, compared with the previous approaches, can be quite improved while allowing rotations for moving objects. Furthermore, they bring up the idea of the MMDT (Modified Morphological Distance Transformation), which combines the rotational morphology for processing the orientation, forward and backward movement into consideration in path planning [4]. Then a better path can be found with low computational complexity.

In [4], for some special end positions, it cannot find the collision-free path by only using the algorithm. So in this paper, we propose an idea of the critical transition point in the previous framework [5] to solve such problem; furthermore, adaptive path planning problems can be solved. Further, we can apply the concept to the 3-D (3-Dimensional) cases so as to be more realistic.

\section{ROTATIONAL MORPHOLOGY AND MMDT}

\subsection{Path Planning by Rotation Morphology}

The concept of the rotational morphology is reviewed as follow

[3]: Assume that the full $360^{\circ}$ angles are equally divided into $N$ parts (or directions). For structuring element $B, B_{i}$ denotes the rotation of $B$ by the degree of $360 i / N$, for $i=0,1, \ldots, N-1$, and let $y$ represent 2-D coordinates. Let $A$ is a binary image and $B$ is a binary structuring element, respectively.

Definition 1: The rotational dilation of $A$ by $B$

$$
\begin{aligned}
(A \tilde{\oplus} B)(\mathrm{y}) & =\left[A \oplus B_{N}-1, A \oplus B_{N}-2, \ldots, A \oplus B_{1}, A \oplus B_{0}\right](\mathrm{y}) \\
& =\left[P_{N}-1, P_{N}-2, \ldots, P_{1}, P_{0}\right](\mathrm{y}) \\
& =\vec{P}(\mathrm{y})
\end{aligned}
$$

where $\vec{P}$ is a $N$-bit codeword matrix.

Definition 2: The rotational erosion of $A$ by $B$

$$
(A \widetilde{\mathrm{e}} B)(\mathrm{y})=\left[A \text { è } B N-1, A \text { è } B N-2, \ldots, A \text { è } B_{1}, A \text { è } B_{0}\right](\mathrm{y})(2)
$$


Fig. 1 shows an example of rotational erosion. The rotational morphology provides not only the geometric hape but also the orientation information. As shown in Fig. 2, the rotational morphology is equivalent to executing $N$ binary morphology modules. The rotational morphology can be computed in parallel; its computational complexity remains the same as the binary morphology. Property 1: The rotational mophology is translation invariant,

$$
\left(A \tilde{\oplus} B_{z}\right)=(A \tilde{\oplus} B)_{z}
$$

where $\mathrm{z}$ denotes any $2-\mathrm{D}$ coordinates.

Property 2: Duality,

$$
A^{c} \tilde{\oplus} \bar{B}=(A \tilde{\mathrm{e}} B)^{c}
$$

where $A^{c}$ represents the complement of set $A$ and $\bar{B}$ represents the symmetric set of $B$ with respect to the origin.

Property 3: Decomposition, for $B=C \cup D$,

$$
\begin{aligned}
& A \tilde{\oplus} B=(A \tilde{\oplus} C) \cup(A \tilde{\oplus} D) \\
& A \tilde{\mathrm{e}} B=(A \tilde{\mathrm{e}} C) \cup(A \tilde{\mathrm{e}} D)
\end{aligned}
$$

\subsection{Modified Morphological Distance Transformation}

When the configuration space is created by rotational morphology to get FP (Free Position), followed by a distance transformation on these regions with the grown obstacles excluded, and then track back the resultant of distance map from the end point through its neighbors with minimum distance until the beginning point is reached. In order to take forward movement and backward movement into consideration at the same time in finding the shortest path, the MMDT is proposed to combine the rotational morphology for processing both the orientations, forward and backward moving information [4]. The new distance transformation algorithm can overcome the drawback that is allowed only for forward movements, as shown in Fig. 3.

As shown in Fig. 4, the distance of the beginning points, which occupied by the VSE (Vehicle-Structuring Element) are set to ' 0 '. The black regions represent the obstacles in the workspace $Z$ Assume that moving object is one of the car-like vehicles, and consider 8 direction model as shown Fig. 3. Then, in order to obtain the candidate points that will propagate from the current point, one can apply morphology dilation with a $3 \times 3$ structuring elment at the driving point of the car-like vehicle marked as current point. To find a collision-free path, we must set this constraint that is not allowed to overlap any pixel of the obstacles and obey all nonphysical constraints; one is their directions must be satisfied with the continuos-turning constraint [3], another is the collision-free requirement. Then distance ' 1 ' is set to these points. The similar procedure can be applied to the corresponding rear points of the driving position so as to obtain the backward propagation candidate points, which is set distance ' -1 ' correspond to one-step backward movement. All the detailed steps of MMDT are in [4].

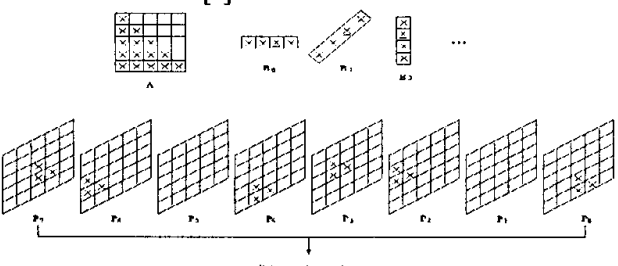

Fig. 1: An example of a rotational erosion operation of $A$ by $B$.
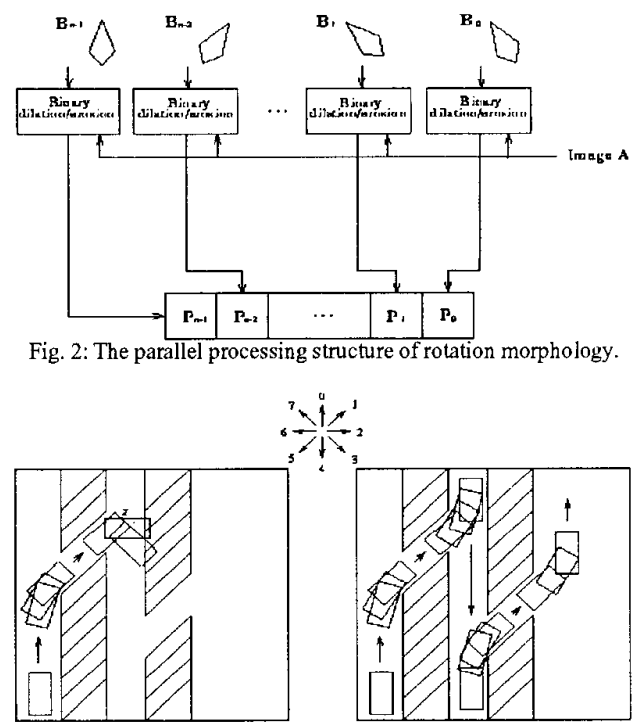

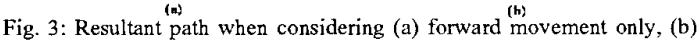
forward and backward movement, into the vehicle model.
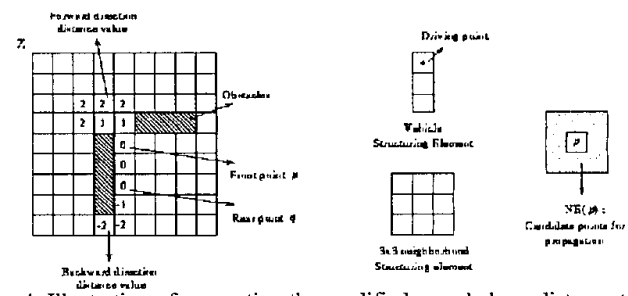

Fig. 4: Illustration of computing the modified morphology distance trans formation for a "p"-shape VSE

\section{THE PROPOSED PATH PLANNING ALGORITHM}

\subsection{Critical Transition Point}

When the distance map is obtaned by MMDT for certain beginning point, we can find that some points are not able to become the end point; however, it may be a feasible end point from another viewpoint. So we modify the original algorithm a little to improve these creumstances.

For some points of the same distance, two different situations will be existed. One is that passing through certain points cause the end point that cannot be arrived from revealing in distance map, but the end point can be actually arrived from other viewpoints. Another is that passing through certain points cause the end point that always cannot be arrived from whatever viewpoints. So starting at the number is smaller in the distance map, the points selected in the path-finding procedure must not only meet continuous turning constraint but also the desired end point can be arrived when it is viewed from another beginning point. This is the concept of the critical transition point. Using back-tracking process to find the optimal path of a reference point which is operated by mophology operations from the beginning point to the critical transition point at first, and the second step is to find the optimal path from the critical transition point to the end point. The 
reason of using back-tracking process is that the fore-tracking process has many choices of the beginning point at first. Then the overall directions of the visited points are encoded as a chain code and the resultant code is sent as the final solution. The detailed algorithm is described as follows:

Step I In a workspace, all points are viewed as the beginning points to create all distance maps made by MMDT.

Step 2. If the desired end point can be arrived for the decided beginning point in a workspace, use back-tracking process directly based on distance map made by MMDT.

Step 3. If it is not met as step 2, repeat step 4 and step 5, and starting at distance value is ' 1 ' to find the critical transition point.

Step 4. For the same value in the distance map, select the point which must not only meet cont inuous turning constraint, but also the desired end point must be arrived from other viewpoints.

Step 5. If step 4 can not be satisfied, the testing value is increasing from ' 1 ' until certain point is satisfied with step 4 , then go to step 6. Otherwise, repeat step 4 and step 5 . If the distance value in the distance map is increasing over maximum in a workspace, stop the procedure. It means no critical transition point exists.

Step 6. When one of the critical transition points is found, use back-tracking process to find the optimal path from the beginning point to the critical transition point at first. And then find the $\varphi-$ timal path from the critical transition point to the end point.

\subsection{Adaptive Path Planning}

If the environment is not known in advance or the environment is changing, path planning must consider this kind of the condition. That is called adaptive path planning. The principle of the solution is to obtain partial information about its environment by viewing it from the present position, and then explore it to gain progressively more information until the desired path can be fully planned. So, based on the concept of the critical transition point adaptive path planning problems can be solved. The manner of adaptive path planning problem is described as below.

Step 1. Create the distance map made by MMDT for a decided beginning point, the object moves one most suitable pace by back-tracking process and critical transition point algorithm.

Step 2. Let the present position be the beginning point; create a new distance map made by MMDT based on moving obstacles. Step 3. Using the new distance map, we decide the next suitable pace of moving object and critical transition point finding.

Step 4. Repeat step 2 and step 3 until the moving object arrives at the desired end point.

Although the moving object can arrive at the end point in a plane (2-Dimensional) in accordance with distance map, we do not guarantee that it has not a collision between the moving object and the obstacles in altitude. So in the path planning in 3-D case, besides considering common the problem of path planning in a plane, we must pay attention to have no collision between them in altitude at the same time. This is the principle of solving path planning in 3-D case.

Roughly speaking, in configuration space method, the significant concept is acquired from these two algorithms joined with 3-D case principle in solving all kinds of path planning problems.

\section{EXPEIRMENTAL RESULTS AND DISCUSSIONS}

In this section, based on two different shape vehicles within a field " $Z$ " with size $11 \times 10$ are examined for illustration. One of the vehicles is a "l" -shape car-like vehicle with length ' 3 ', the other is non-symmetrical vehicle of " $L$ " -shape with length ' 3 ',

As described above, we find that the end point $(7,10)$ cannot be arrived when the beginning point is $(3,5)$ with " $L$ " -shape car-like vehicle, as shown in the distance map of Fig. 5 . All outcomes of the simulation include the distance map and the results of back-tracking. In Fig. 5 and Fig. 6, we can find that when using the concept of the critical transition point, the point $(7,10)$ which can not be arrived shown in Fig. 5 will be arrived, the beginning point is still $(3,5)$ (or the beginning point is $(5,5)$ for backward movement). And the critical transition point is $(6,5)$ in this case (or the critical transition point is $(8,5)$ for backward movement). There are two circumstances that we must emphasize. One is in considering forward and backward path planning, each one of the front driving point (the upper part of the moving object) and the rear driving point (the lower of the moving object, i.e. its reverse point) takes as ' $\mathrm{s}$ ' in figure (the beginning point). Because the difference between the two cases is just forward movement and backward movement at first, the way of doing is reasonable. The other is in " $\mathrm{L}$ "-shape car-like vehicle, any a part of moving object arrive the desired end point, not necessarily the driving point, we think the path-finding to be legitimate, shown as in Fig. 6.

The simulation of adaptive path planning is from Fig. 7 to Fig. 10. In the first half of the phase, the vehicle is "|"-shape car-like vehicle with length 3 . Figs. 7 and 8 represent the shortest path which contains the concept of the critical transition point when the environment is shown as the figure, where the beginning point is $(9,4)$ and the end point is $(7,10)$. If the environment is changing as shown in Fig. 9 (or Fig. 10) after transiting a pace of moving dject in Fig. 7, the optimal path is shown as Fig. 9 and Fig. 10, which also contains the concept of the critical transition point. Worth to mention is the distinct results between two environments. It stands for the meaning of "adaptive".

\section{CONCLUSION}

To find the shortest collision-free path among obstacles for arbitrary vehicle model, some general algorithms for solving such problem utilizing the rotational morphology and the modified morphological distance transformation are proposed in this paper. The concept of the critical transition point can solve the problem of the desired end point that can not be arrived originally in previous works. The manner of adaptive path planning can find the shortest path adaptively at any time even though the environment is always changing. However, to join in 3-D case principle of the path planning, it is more conformed to the circumstance of the real world. Note that these two algorithms do not increase the computational complexity compared to previous algorithms.

\section{REFERENCES}

[1] P. L. Lin and S. Chang, "A shortest path algorithm for a nonrotating object among obstacles of arbitrary shapes," IEEE Trans. Syst., Man, Cybern., vol. 23, pp. 825-833, 1993.

[2] O. Takahashi and R. J. Schiling, "Motion planning in a plane using generalized Voronoi diagrams," IEEE Trans. Robot. Automat, vol. 6, pp. 143-150, Apr. 1989.

[3] S. C. Pei, C. L. Lai, and F. Y. Shih, "A morphological approach to shortest path planning for rotating objects," Pattern Recognition, vol. 31, no. 8, pp. 1127-1138, Aug. 1998.

[4] S. C. Pei, C. L. Lai, "Forward and backward motion planning for rotating objects based on morphology," $I I^{\text {th }}$ IPPR Conference on Comput. Vision, Graphics, Image Processing, pp. 346-353, Wu-Lai, Taiwan, ROC, Aug. 1998

[5] T. Lozano-Perez and M. A. Wesley, "An algorithm for pla nning collision-free paths among polyhedral obstacles," Communication of the $A C M$, vol. 22, no. 10, pp. 550-570, 1979. 


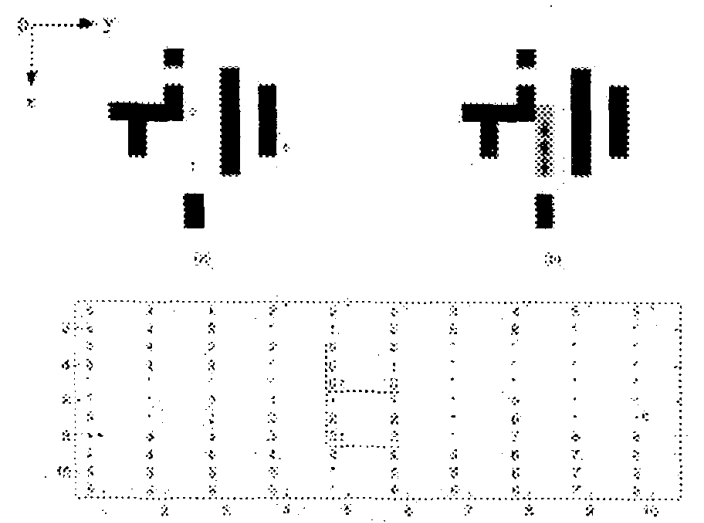

Fig. 5: Simulation results of the critical transition point for "L"-shape VSE. Note that when the beginning point is $(5,5)$, the critical transition point is $(8,5)$ for backward movement, and the desired end point $(7,10)$ can not be arrived. Chain code is [4 4 4].

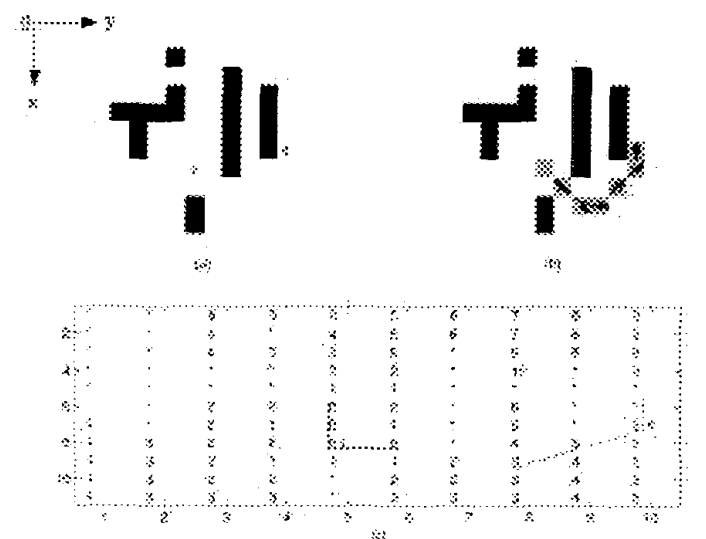

Fig. 6: (Continued from Fig. 5), the critical transition point is $(8,5)$ for backward movement and the end point is $(7,10)$. The chain code of the resultant path is $\left[\begin{array}{llllll}3 & 3 & 2 & 1 & 1 & 0\end{array}\right]$.
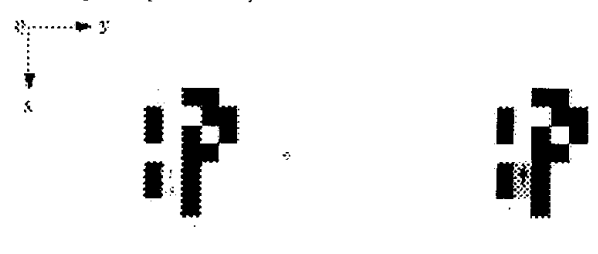

w:

14

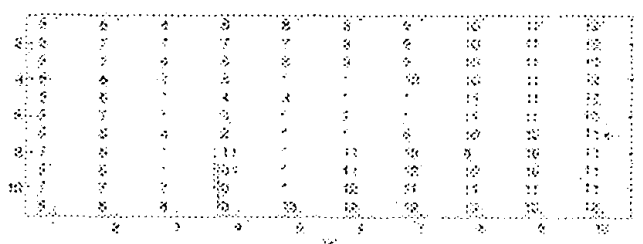

Fig. 7: Simulation results of adaptive motion planning for "."-shape VSE. Note that the beginning point is $(9,4)$, the critical transition point is $(8,4)$ for forward movement. Chain code is [0].


Fig. 8: (Continued from fig. 7), the critical transition point is $(8,4)$ for forward movement and the end point is $(7,10)$. The chain code of the resultant path is $[00000733321]$.
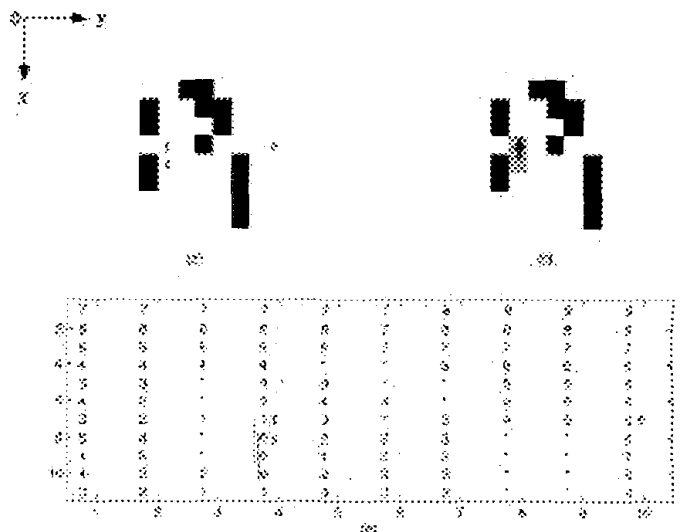

Fig. 9: (Continued from Fig. 7), the environment is changing after transiing a pace of VSE. Note that the beginning point is $(8,4)$, the critical transition point is $(7,4)$ for forward movement. Chain code is $[0]$.


Fig. 10: (Continued from Fig. 9), the critical transition point is $(7,4)$, but we start from another beginning point $(9,4)$ for backward movement, and the end point is $(7,10)$. The chain code of the resultant path is $\left[\begin{array}{lllll}5 & 2 & 2 & 1 & 2\end{array}\right]$. 